\title{
Fractional exponential operators and time-fractional telegraph equation
}

Alireza Ansari*

\section{*Correspondence:}

alireza_1038@yahoo.com

Department of Applied

Mathematics, Faculty of

Mathematical Sciences, Shahrekord

University, P.O. Box 115, Shahrekord, Iran

\begin{abstract}
In this paper, the Bromwich integral for the inverse Mellin transform is used for finding an integral representation for a fractional exponential operator. This operator can be considered as an approach for solving partial fractional differential equations. Also, application of this operator for obtaining a formal solution of the time-fractional telegraph equation is discussed.
\end{abstract}

MSC: 26A33; 35A22; 44A10

Keywords: Laplace transform; Mellin transform; partial fractional differential equation; Wright function

\section{Introduction and problem}

We consider the exponential operator

$$
e^{\lambda\left[q(x) \frac{d}{d x}+v(x)\right]} f(x)=f(x(\lambda)) g(\lambda)
$$

where $x(\lambda), g(\lambda)$ are specified by the system of first-order differential equations [1]

$$
\left\{\begin{array}{l}
\frac{d}{d \lambda} x(\lambda)=q(x(\lambda)), \quad x(0)=x \\
\frac{d}{d \lambda} g(\lambda)=v(x(\lambda)) g(\lambda), \quad g(0)=1 .
\end{array}\right.
$$

By the above exponential operator, Dattoli et al. found solutions of some boundary value problems arising in mathematical physics in terms of integral transforms type; see [2, 3] and references therein. Also, they used this operational technique to describe properties of some special polynomials and functions [4-6]; also see [4].

When we encounter an exponential operator of higher order $e^{\lambda^{\alpha} s^{\alpha}}$, where $\alpha$ is integer or non-integer and $s=q(x) \frac{d}{d x}+v(x)$, it is of interest to have an integral representation to reduce the order and apply the relation (1.1). For example, for exponential operators of orders two and three, we can write the Gauss-Weierstrass and the Airy integrals [2, 7]

$$
\begin{aligned}
e^{\lambda^{2} s^{2}} & =\frac{1}{\sqrt{\pi}} \int_{-\infty}^{\infty} e^{-\xi^{2}+2 \lambda s \xi} d \xi, \\
e^{\lambda^{3} s^{3}} & =\int_{-\infty}^{\infty} e^{\sqrt[3]{3} \lambda s \xi} \operatorname{Ai}(\xi) d \xi,
\end{aligned}
$$

(c) 2012 Ansari; licensee Springer. This is an Open Access article distributed under the terms of the Creative Commons Attribution License (http://creativecommons.org/licenses/by/2.0), which permits unrestricted use, distribution, and reproduction in any medium, provided the original work is properly cited. 
where $\operatorname{Ai}(\xi)$ is the Airy function of the first kind given by

$$
\operatorname{Ai}(\xi)=\frac{1}{\pi} \int_{0}^{\infty} \cos \left(\frac{t^{3}}{3}+\xi t\right) d t
$$

For the fractional exponential operator $e^{-\lambda^{\alpha} s^{\alpha}}, 0<\alpha<1$, it may occur that this operator can be written as the Laplace transform of the Wright function [8-10]

$$
e^{-\lambda^{\alpha} s^{\alpha}}=\int_{0}^{\infty} e^{-s t} \frac{1}{t} W\left(-\alpha, 0 ;-\lambda^{\alpha} t^{-\alpha}\right) d t
$$

where the Wright function is presented by the following relation [11]:

$$
W(\alpha, \beta ; t)=\sum_{k=0}^{\infty} \frac{t^{k}}{k ! \Gamma(\alpha k+\beta)}, \quad \alpha>-1, \beta \in \mathbb{C}, t \in \mathbb{C} .
$$

In this paper, in a general case we obtain an integral representation for $e^{\lambda^{\alpha} s^{\alpha}}, \alpha>0$, with order one for $s$, and then we show how this operator can be applied to find the formal solutions of partial fractional differential equations (PFDEs).

This problem for integral representation is referred to as the inverse of the Mellin transform of $e^{\lambda^{\alpha} s^{\alpha}}, \alpha>0$, and in Section 2, we state main theorems and corollaries related to it. In Section 3, as an application of this technique, we find formal solutions of the spacefractional Moshinskii's equation and the time-fractional telegraph equation. Finally, in Section 4 the main conclusions are drawn.

\section{Main theorems and corollaries}

In this section, we establish some theorems on the fractional exponential operator which can be useful for solving PFDEs. First, we derive an integral representation for the operator $e^{\lambda^{n} s^{n}}$, which can be considered as a generalized representation of the relations (1.3) and (1.4).

Theorem 2.1 The following identity holds true for $c_{1}<\Re s<c_{2}$ :

$$
e^{\lambda^{n} s^{n}}=\frac{1}{\pi} \int_{-\infty}^{\infty} e^{s \xi} \mathcal{A}_{n}(\xi, \lambda) d \xi, \quad n=2,3, \ldots
$$

where the function $\mathcal{A}_{n}(\xi, \lambda)$ is presented by

$$
\mathcal{A}_{n}(\xi, \lambda)= \begin{cases}\left.\int_{0}^{\infty} \cos \left(r \xi+(-1)^{\frac{n+1}{2}} \lambda^{n} r^{n}\right)\right) d r, & n=2 k+1, \\ \frac{1}{n \lambda} \int_{0}^{\infty} e^{-r} \frac{\cos \left(\frac{1}{\lambda} r^{\frac{1}{n}} \xi\right)}{r^{1-\frac{1}{n}}} d r, & n=4 k+2, \\ \frac{1}{n \lambda} \int_{0}^{\infty} e^{r} \frac{\cos \left(\frac{1}{\lambda} r^{\frac{1}{n}} \xi\right)}{r^{1-\frac{1}{n}}} d r, & n=4 k .\end{cases}
$$

Proof By the definition of the inverse of the Mellin transform for a function $e^{\lambda^{n} s^{n}}$, we have

$$
\begin{aligned}
\frac{1}{2 \pi i} \int_{c-i \infty}^{c+i \infty} e^{\lambda^{n} s^{n}} t^{-s} d s & =\frac{1}{2 \pi} \int_{0}^{\infty} e^{(-i \lambda r)^{n}} t^{i r} d r+\frac{1}{2 \pi} \int_{0}^{\infty} e^{(i \lambda r)^{n}} t^{-i r} d r \\
& =\frac{1}{2 \pi} \int_{0}^{\infty} e^{\lambda^{n} r^{n} e^{-\frac{n \pi}{2} i}} t^{i r} d r+\frac{1}{2 \pi} \int_{0}^{\infty} e^{\lambda^{n} r^{n} e^{\frac{n \pi}{2}} i} t^{-i r} d r
\end{aligned}
$$




$$
\begin{aligned}
= & \frac{1}{2 \pi} \int_{0}^{\infty} e^{\lambda^{n} r^{n} \cos \left(\frac{n \pi}{2}\right)} e^{-i \lambda^{n} r^{n} \sin \left(\frac{n \pi}{2}\right)+i r \ln (t)} d r \\
& +\frac{1}{2 \pi} \int_{0}^{\infty} e^{\lambda^{n} r^{n} \cos \left(\frac{n \pi}{2}\right)} e^{i \lambda^{n} r^{n} \sin \left(\frac{n \pi}{2}\right)-i r \ln (t)} d r \\
= & \frac{1}{\pi} \int_{0}^{\infty} e^{\lambda^{n} r^{n} \cos \left(\frac{n \pi}{2}\right)} \cos \left(r \ln (t)-\lambda^{n} r^{n} \sin \left(\frac{n \pi}{2}\right)\right) d r
\end{aligned}
$$

The above relation implies that the Mellin transform of the last integral is equal to the function $e^{\lambda^{n} s^{n}}$, that is,

$$
\begin{aligned}
e^{\lambda^{n} s^{n}} & =\frac{1}{\pi} \mathcal{M}\left\{\int_{0}^{\infty} e^{\lambda^{n} r^{n} \cos \left(\frac{n \pi}{2}\right)} \cos \left(r \ln (t)-\lambda^{n} r^{n} \sin \left(\frac{n \pi}{2}\right)\right) d r ; s\right\} \\
& =\frac{1}{\pi} \int_{0}^{\infty} t^{s-1} \int_{0}^{\infty} e^{\lambda^{n} r^{n} \cos \left(\frac{n \pi}{2}\right)} \cos \left(r \ln (t)-\lambda^{n} r^{n} \sin \left(\frac{n \pi}{2}\right)\right) d r d t
\end{aligned}
$$

By setting $\ln (t)=\xi$, we get the relation (2.1).

Theorem 2.2 (The Schouten-Van der Pol theorem for the Laplace transform [12]) Let c be a suitable real constant such that $F(s)$ and $\Phi(s)$ are analytic functions in the half-plane $\Re s>c$ and $F(s)$ is the Laplace transform off $(t)$. Then the inverse of the Laplace transform $F(\Phi(s))$ is given by

$$
g(t)=\mathcal{L}^{-1}\{F(\Phi(s)) ; t\}=\int_{0}^{\infty} f(\tau)\left[\frac{1}{2 \pi i} \int_{c-i \infty}^{c+i \infty} e^{-\Phi(s) \tau+t s} d s\right] d \tau
$$

Proof Using the definition of the Laplace transform for $F(\Phi(s))$

$$
F(\Phi(s))=\int_{0}^{\infty} e^{-\Phi(s) \tau} f(\tau) d \tau
$$

replacing in the inverse of the Laplace transform $F(\Phi(s))$

$$
g(t)=\mathcal{L}^{-1}\{F(\Phi(s)) ; t\}=\frac{1}{2 \pi i} \int_{c-i \infty}^{c+i \infty} F(\Phi(s)) e^{t s} d s
$$

and changing the order of integration, we get the relation (2.4).

Corollary 2.3 It is obvious that by setting $\Phi(s)=s^{\alpha}, 0<\alpha<1$, in the relations (2.4) and using the relation (1.6) for the inverse of the Laplace transform $e^{-s^{\alpha}}$, the inverse of the Laplace transform $F\left(s^{\alpha}\right)$ can be presented by

$$
\mathcal{L}^{-1}\left\{F\left(s^{\alpha}\right) ; t\right\}=\frac{1}{t} \int_{0}^{\infty} f(\tau) W\left(-\alpha, 0 ;-\tau t^{-\alpha}\right) d \tau .
$$

Corollary 2.4 By setting $F(s)=e^{\lambda^{n} s^{n}}$ and combining the relations (2.1) and (2.5), we get a new integral representation for the fractional exponential equation $e^{\lambda^{n} s^{\gamma}}$

$$
e^{\lambda^{n} s^{\gamma}}=\frac{1}{\pi} \int_{-\infty}^{\infty} e^{s \xi} \mathcal{A}_{n}^{(\alpha)}(\xi, \lambda) d \xi, \quad \gamma=n \alpha, 0<\alpha \leq 1
$$


where the function $\mathcal{A}_{n}^{(\alpha)}(\xi)$ is given by

$$
\mathcal{A}_{n}^{(\alpha)}(\xi, \lambda)=\frac{1}{\xi} \int_{0}^{\infty} \mathcal{A}_{n}(\tau, \lambda) W\left(-\alpha, 0 ;-\tau \xi^{-\alpha}\right) d \tau .
$$

In view of the theorems of a fractional exponential operator expressed in this section, we may apply this operator to PFDEs in the next section.

\section{Application to partial fractional differential equations}

Example 3.1 In connection with initial-value diffusions, we consider the space-fractional Moshinskii's equation of order $\gamma$ in the Riemann-Liouville sense [12]

$$
\frac{\partial}{\partial t} u(x, t)=\frac{1}{1+x^{2}}\left(\left(1+x^{2}\right) \frac{\partial}{\partial x}\right)^{\gamma} u(x, t), \quad \gamma>0, x>0, t>0
$$

with the Cauchy-type initial condition as $u(x, 0)=f(x)$.

In order to obtain the solution of (3.1), by solving the first-order partial differential with respect to $t$ and applying the initial condition, the formal solution in the form of fractional exponential operator gives rise to

$$
u(x, t)=e^{\frac{t}{1+x^{2}}\left(\left(1+x^{2}\right) \frac{\partial}{\partial x}\right)^{\gamma}} f(x) .
$$

Now, by setting $\left(\frac{t}{1+x^{2}}\right)^{\frac{1}{n}}=\lambda, s=\left(1+x^{2}\right) \frac{\partial}{\partial x}$ and applying Corollary 2.4 for the integral representation of $e^{\lambda^{n} s^{\gamma}}$, we can write the solution in terms of the integral transform as

$$
u(x, t)=\frac{1}{\pi} \int_{-\infty}^{\infty} e^{\left(1+x^{2}\right) \frac{\partial}{\partial x} \xi} \mathcal{A}_{n}^{(\alpha)}(\xi, \lambda) f(x) d \xi, \quad \gamma=n \alpha, 0<\alpha \leq 1,
$$

where the function $\mathcal{A}_{n}^{(\alpha)}(\xi, \lambda)$ is given by the relation (2.7). The above relation can be simplified in the following form:

$$
u(x, t)=\frac{1}{\pi} \int_{-\infty}^{\infty} \mathcal{A}_{n}^{(\alpha)}\left(\xi,\left(\frac{t}{1+\left(\tan \left(\xi+\tan ^{-1}(x)\right)\right)^{2}}\right)^{\frac{1}{n}}\right) f\left(\tan \left(\xi+\tan ^{-1}(x)\right)\right) d \xi,
$$

where we used the relations (1.1) and (1.2) by choosing the functions $q(x)=1+x^{2}$ and $v(x)=0$.

Example 3.2 As another application, we consider the time-fractional telegraph equation $[13,14]$

$$
\frac{\partial^{2}}{\partial x^{2}} u(x, t)=\left[a \frac{\partial^{2 \alpha}}{\partial t^{2 \alpha}}+b \frac{\partial^{\alpha}}{\partial t^{\alpha}}+c\right] u(x, t), \quad 0<\alpha \leq 1, x, t>0, a, b, c \in \mathbb{R}
$$

with initial and asymptotic conditions $u(0, t)=f(t), \lim _{x \rightarrow \infty} u(x, t)=0$.

Similar to the previous problem by solving the equation with respect to $x$ and applying the initial and asymptotic conditions, the formal solution takes the form:

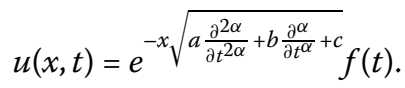


Now, by setting $\frac{\partial}{\partial t}=s$ and writing an integral representation for $e^{-x \sqrt{a s^{2}+b s+c}}$ in terms of the Bessel function of order one, we get [15]

$$
\begin{aligned}
& e^{-x \sqrt{a s^{2}+b s+c}} \\
& \quad=e^{-x\left(\frac{b}{2 \sqrt{a}}+\sqrt{a s}\right)}-x \sqrt{\frac{\Delta}{a}} \int_{x \sqrt{a}}^{\infty} e^{-s u-\frac{b}{2 a}} \frac{J_{1}\left(\sqrt{\frac{\Delta}{a}} \sqrt{u^{2}-a x^{2}}\right)}{\sqrt{u^{2}-a x^{2}}} d u, \quad \Delta=a c-\frac{b^{2}}{4} \neq 0 .
\end{aligned}
$$

We can rewrite the relation (3.5) in the following form:

$$
\begin{aligned}
u(x, t)= & e^{-\frac{x b}{2 \sqrt{a}}} \int_{0}^{\infty} \frac{1}{\tau} W\left(-\alpha, 0 ;-x \sqrt{a} \tau^{-\alpha}\right) f(t-\tau) d \tau \\
& -x \sqrt{\frac{\Delta}{a}} e^{-\frac{b}{2 a}} \int_{0}^{\infty} \frac{1}{\tau} f(t-\tau) \int_{x \sqrt{a}}^{\infty} \frac{J_{1}\left(\sqrt{\frac{\Delta}{a}} \sqrt{u^{2}-a x^{2}}\right)}{\sqrt{u^{2}-a x^{2}}} \\
& \times W\left(-\alpha, 0 ;-u \tau^{-\alpha}\right) d u d \tau,
\end{aligned}
$$

where we used the relation (2.6) for the linearization of a fractional exponential operator $e^{-x s^{\alpha}}$, and then we applied the relations (1.1) and (1.2) by substituting $q(x)=1$ and $v(x)=0$.

\section{Conclusions}

This paper provides some new results in the theory of fractional derivative. These results show the flexible operational technique can be used in a fairly wide context beside the integral transforms for obtaining the formal solutions of PFDEs.

Also, this technique can be considered as a promising approach for many applications in applied sciences.

\section{Competing interests}

The author declares that he has no competing interests.

\section{Acknowledgements}

The author was partially supported by the Center of Excellence for Mathematics, University of Shahrekord.

Received: 27 July 2012 Accepted: 12 October 2012 Published: 29 October 2012

\section{References}

1. Dattoli, G, Ottaviani, PL, Torte, A, Vazquez, L: Evolution operators equations: integration with algebraic and finite difference methods. Applications to physical problems in classical and quantum mechanics and quantum field theory. Riv. Nuovo Cimento 20, 1-133 (1997)

2. Dattoli, G, Srivastava, HM, Zhukovsky, Z: Operational methods and differential equations with applications to initial-value problems. Appl. Math. Comput. 184, 979-1001 (2007)

3. Dattoli, G, Ricci, PE, Khomasuridze, I: Operational methods, special polynomial and functions and solution of partial differential equations. Integral Transforms Spec. Funct. 15(4), 309-321 (2004)

4. Babusci, D, Dattoli, G, Sacchetti, D: The Airy transform and the associated polynomials. Cent. Eur. J. Phys. 9(6), 1381 (2011)

5. Dattoli, G: Operational methods, fractional operators and special polynomials. Appl. Math. Comput. 141, 151-159 (2003)

6. Dattoli, G, Ricci, PE, Sacchetti, D: Generalized shift operators and pseudo-polynomials of fractional order. Appl. Math. Comput. 141, 215-224 (2003)

7. Vallee, O, Soares, M: Airy Functions and Applications to Physics. Imperial College Press, London (2004)

8. Aghili, A, Ansari, A: Solving partial fractional differential equations using the $\mathcal{L}_{A}$-transform. Asian-Eur. J math. 3(2), 209-220 (2010)

9. Aghili, A, Ansari, A: Solution to system of partial fractional differential equation using the $\mathcal{L}_{2}$-transform. Anal. Appl. 9(1), 1-9 (2011)

10. Ansari, A, Refahi Sheikhani, A, Saberi Najafi, H: Solution to system of partial fractional differential equation using the fractional exponential operators. Math. Methods Appl. Sci. 35, 119-123 (2012)

11. Kilbas, AA, Srivastava, HM, Trujillo, JJ: Theory and Applications of Fractional Differential Equations. North-Holland Mathematics Studies, vol. 204. Elsevier, Amsterdam (2006) 
12. Duffy, DG: Transform Methods for Solving Partial Differential Equations. CRC Press, New York (2004)

13. Chen, J, Liu, F, Anh, V: Analytical solution for the time-fractional telegraph equation by the method of separating variables. J. Math. Anal. Appl. 338, 1364-1377 (2008)

14. Orsingher, E, Beghin, L: Time-fractional telegraph equations and telegraph processes with Brownian time. Probab. Theory Relat. Fields 128, 141-160 (2004)

15. Ditkin, VA, Prudnikov, AP: Integral Transforms and Operational Calculus. Pergamon, Elansford (1965). (English translation)

doi:10.1186/1687-2770-2012-125

Cite this article as: Ansari: Fractional exponential operators and time-fractional telegraph equation. Boundary Value Problems 2012 2012:125.

Submit your manuscript to a SpringerOpen ${ }^{\odot}$ journal and benefit from:

- Convenient online submission

- Rigorous peer review

- Immediate publication on acceptance

- Open access: articles freely available online

- High visibility within the field

- Retaining the copyright to your article 